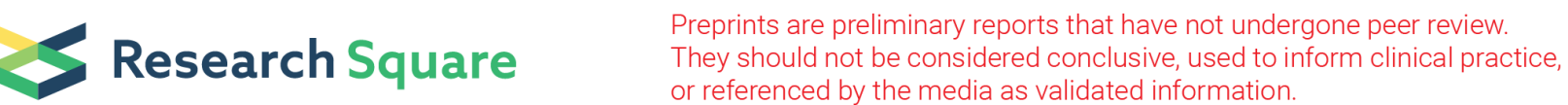

\section{Deworming women of reproductive age during adolescence and pregnancy: what is the impact on morbidity from soil-transmitted helminths infection?}

Carolin Vegvari ( $\nabla$ c.vegvari@imperial.ac.uk)

https://orcid.org/0000-0003-2018-011X

Federica Giardina

Erasmus MC

Sumali Bajaj

Imperial College London

Veronica Malizia

Erasmus MC

Robert J. Hardwick

Imperial College London https://orcid.org/0000-0001-8778-006X

James E Truscott

Imperial College London https://orcid.org/0000-0002-2555-7636

Antonio Montresor

World Health Organization https://orcid.org/0000-0003-3103-8872

Sake $\mathrm{J}$ de Vlas

Erasmus MC https://orcid.org/0000-0002-1830-5668

Luc E Coffeng

Erasmus MC https://orcid.org/0000-0002-4425-2264

Roy M Anderson

Imperial College London

Research

Keywords: Reproductive, Soil-transmitted helminths, neglected tropical diseases

Posted Date: January 5th, 2021

DOI: https://doi.org/10.21203/rs.3.rs-65385/v2

License: (c) (1) This work is licensed under a Creative Commons Attribution 4.0 International License.

Read Full License 
Version of Record: A version of this preprint was published at Parasites \& Vectors on April 23rd, 2021. See the published version at https://doi.org/10.1186/s13071-021-04620-w. 


\section{Abstract}

Background: Soil-transmitted helminths (STHs) are a major cause of poor health in low- and middleincome countries. In particular, hookworm is known to cause anaemia in children and women of reproductive age (WRA). One goal of the World Health Organization's (WHO) 2030 roadmap for neglected tropical diseases is to reduce STH-related morbidity in WRA. As a minimal intervention the WHO recommends deworming adolescent girls annually during human papilloma virus vaccination programmes and women of reproductive age (WRA) during pregnancy and lactation. These routine interventions are low cost can be implemented even from the most basic health services in endemic countries. In this study we use a cohort model to investigate the potential impact on STH-related morbidity in WRA.

Results: Annual deworming treatment of adolescent girls reduces the prevalence of M\&HI infections in this age group by up to $60 \%$ in moderate transmission settings and by $12-27 \%$ in high-transmission settings. Treatment of WRA during pregnancy and lactation on its own has a small but significant effect on morbidity although it does not lead to the achievement of the morbidity target ( $<2 \%$ moderate-to-high intensity infections) in this age group. However, depending on the age-intensity profile of infection, which may vary geographically, and assumptions on the density-dependence of egg production by fertilised female worms, continued school-based treatment may be able to reduce the force of infection acting on WRA, both through an indirect effect on the overall population based force of infection and via reducing the burden of infection as children age and move into the WRA age classes. As a result, morbidity in WRA may be eliminated.

Conclusion: While deworming during pregnancy and lactation does not lead to the achievement of the morbidity target in WRA and its efficacy may vary by setting, it is still expected to be beneficial for maternity and child health. Monitoring of any WRA-based intervention is recommended to evaluate its effectiveness.

\section{Background}

Soil-transmitted helminths (STH) are tropical parasites that affect 1.7 billion people worldwide [1]. Hookworm (Ancylostoma duodenale and Necator americanus) in particular can cause significant morbidity, mainly anaemia. The severity of anaemia has been observed to increase in individuals with moderate-to-high intensity (M\&HI) infection [2,3]. In hookworm, M\&HI infection is defined as more than 2000 eggs per gram (epg) faeces measured by a Kato-Katz faecal smear test [4]. Anaemia can lead to stunted development during childhood, has a negative impact on the health of women of reproductive age (WRA) and can contribute to adverse pregnancy outcomes [3]. Current treatment guidelines recommend school-based (SB) deworming of pre-school age children (pSAC, 2-4 years) and school-age children (SAC, 5-14 years) through mass drug administration (MDA), which is given annually in moderateprevalence settings (20-50\%) and semi-annually in high-prevalence settings $(>50 \%)$. In addition, as part of the new 2030 roadmap to achieve morbidity control of STH, the WHO recommends the treatment of WRA 
(18 - 49 years) and adolescent girls (15 - 19 years) [5]. Benzimidazole drugs for the treatment of STHs are currently only donated for pSAC and SAC, but not for adults. In addition, costs of a community wide distribution can be significant. Therefore, community-wide treatment of WRA is not economically feasible for most low- and middle-income countries (LMICs) with the presently available resources.

To minimise the most adverse impact of anaemia on WRA during pregnancy and lactation, the WHO proposes an economically feasible strategy to treat WRA when they come into contact with the health system at prenatal and postnatal clinics. In addition, the WHO recommends preventive chemotherapy (PC) treatment of adolescent girls during annual human papilloma virus (HPV) vaccine programmes. The rationale behind this strategy is that after years of SB treatment, the prevalence of M\&HI infections in children/adolescents is low, on average $2 \%$ after five years of MDA in a review of studies from 17 countries [6]. Consequently, young women in most countries, where annual SB deworming is implemented at effective coverage ( $75 \%$ for STHs), are expected to start their reproductive lifespan with no or low worm burden so that young girls leaving school do not immediately require MDA. The hypothesis is that infection intensity needs time to build up and that treating adolescent girls and WRA (new strategy), who have passed through SB-deworming as children, at regular intervals during pregnancy and lactation could prevent them from developing M\&HI infections. We use a simple cohort model and the force of infection (Fol) derived from two independently developed individual-based stochastic models of STH infection to investigate this hypothesis. The Fol is the per capita rate at which susceptible individuals acquire worms [7]. We choose a cohort model because it makes it easier to follow a cohort of women under controlled conditions compared to the fully stochastic individual-based models which include additional complexities. A cohort model is computationally simpler and fulfils the purpose of our analysis equally well. We focus on hookworm because hookworm-induced anaemia is a recognised cause of pregnancy morbidity and mortality in LMICs and the relationship of infection intensity and morbidity is better understood and documented than for other STHs [8].

\section{Methods}

\section{Cohort model}

We use a cohort model that compares the currently implemented strategy (SB treatment of pSAC and SAC) and the new strategy that extends deworming to adolescent girls and WRA during pregnancy and lactation. We simulate a birth cohort of girls/women and express the effectiveness of the new strategy as the reduction of the risk to develop morbidity in adolescent girls and WRA. In the cohort model all girls/women are simulated from age 0 years and are followed up to age 70. Other individuals are not simulated in the cohort model. We apply external Fols to the cohort of girls/women. The reason is that the Fol cannot be inferred from the cohort model itself because the reservoir of infection for STH transmission is contributed to by the entire community, and not just the cohort of interest. The Fols have been extracted from two different individual-based stochastic transmission dynamics models of STH infection developed at Imperial College London (ICL) $[9,10]$ and Erasmus MC (EMC), Rotterdam [11]. The cohort model itself does not take into account reductions in the Fol due to treating WRA. This assumption 
is based on the consideration that not all women are pregnant at the same time, and even if $80 \%$ of pregnant women visit maternity health centres and receive deworming treatment, this corresponds to very low annual adult coverage that would not contribute much to depleting the infectious reservoir.

\section{Cohort model assumptions}

The cohort model is based on the following assumptions:

- Women with M\&HI infections suffer morbidity from hookworm infection.

- SB treatment only happens when women are aged 2-14 years.

- Coverage of $75 \%$ for PSAC and SAC. Non-access to treatment is assumed to be random at an individual level, i.e., independent of an individual's infection status and history of participating in deworming.

- Deworming of adolescent girls (15-19 years) happening through annual SB programmes and during HPV vaccination programmes. It is assumed that annual HPV programmes will target the whole cohort of girls aged 15-19, and although each adolescent girl is only vaccinated once, all girls of the cohort will attend the programme. This gives an opportunity to treat adolescent girls for STH on an annual basis. The assumed treatment coverage in this cohort is $75 \%$. Non-access to treatment is assumed to be random.

- Deworming during pregnancies occurs twice, during the second and third trimester, at prenatal clinics. Furthermore, we assume on average four pregnancies per woman over the reproductive lifespan (15-50 years of age) [12]. Deworming happens one more time during the year after pregnancy at maternity health clinics. Further, $80 \%$ of pregnant and lactating women make use of prenatal and postnatal clinics. This figure is based on estimated coverage of pregnant women through existing health services [13]. Access to maternity services and to treatment are assumed to be random at the level of the individual.

\section{Stochastic, individual-based model}

Details of the two transmission dynamics models have been published previously $[9,11,14]$. Unlike the cohort model, the fully stochastic individual-based STH transmission models represent age-structured human populations. The age structure in both models follows demographic data from Kenya taken from the 2003 Demographic and Health Surveys [15]. Each simulated year, pSAC and SAC receive SB deworming treatment. Each transmission dynamics model was run 100 times, the Fols were estimated in monthly timesteps and the mean Fols of 100 runs over time were applied to the cohort model.

There are two important differences between the ICL and the EMC model. Firstly, the ICL model assumes that the relative exposure to hookworm infections and contribution to the environmental reservoir of infection are the same for all age groups and only depend on the burden of adult worms in an age group [16]. In other words, the Fol is fixed and independent of age. In contrast, the EMC model assumes that the relative exposure and contribution to the environmental reservoir increase linearly from age 0 years until 
age 10 years and stay constant thereafter [8]. Secondly, both models assume density-dependent saturation of egg production by female hookworm inside the human host. The ICL model assumes that this saturation follows an exponential function $[9,17]$, whereas the EMC model assumes a hyperbolic saturation function [11]. The distinction between these two being that individuals with large worm burdens in the ICL model contribute much smaller egg/larvae counts into the reservoir of infection than those in the EMC model. Due to the sparse and high-variance data to infer the tails of these egg production functions, the difference between the ICL and EMC models remains an unavoidable modelling uncertainty that necessitates a comparison of the conclusions drawn with both for greater scientific rigor.

\section{Scenarios simulated using the cohort model}

We apply Fols extracted from the two fully stochastic individual-based STH transmission models where ongoing annual or semi-annual SB treatment has been implemented over the whole time-course of the simulation, 70 years, to obtain a Fol value under treatment for each year of the girls'/women's lifespan. However, the girls in the cohort only receive SB-treatment when they are pSAC or SAC themselves.

We focus on two SB treatment strategies: annual and semi-annual MDA targeting PSAC and SAC for hookworm species. Annual SB treatment is recommended in settings where the prevalence of infection with any STH species is $20-50 \%$ (moderate). Semi-annual SB treatment is implemented in settings where the prevalence of infection with any STH species is $>50 \%$ (high). We simulate semi-annual treatment for settings with high hookworm prevalence. For settings with moderate prevalence we simulate both annual and semi-annual treatment. This is because although the prevalence of hookworm alone may be moderate, the prevalence of all infections, including other STH species, may be high.

For each strategy, we then compare SB treatment only and SB treatment plus treatment of WRA according to the new suggested guidelines (annual treatment of adolescent girls, treatment twice during pregnancy and once during lactation, irrespective of infection prevalence). Each simulated scenario of the cohort model is run for 100 iterations and the mean prevalence of M\&HI infections over time and $95 \%$ credible intervals are calculated from the results. Each iteration is run with a seed in a reproducible way so that each iteration of the SB treatment only scenario pairs with an iteration of the SB plus WRA treatment scenario.

The average prevalence of M\&HI infections over all simulated time points for adolescent girls and WRA is calculated for each scenario. Table 1 lists all scenarios considered as part of this study. The code for the cohort model can be found in the github repository: https://github.com/caro-veg/STH_WRA_Morbidity. The parameters that are used in the stochastic individual-based simulations to generate the Fols are included in Additional File 1.

The relative reduction in the prevalence of $\mathrm{M} \& \mathrm{HI}$ infections is calculated in the following way: 


\section{Statistical comparison of SB treatment only vs SB treatment plus WRA treatment}

The average point prevalences for adolescent girls, WRA and adolescent girls combined with WRA are compared between paired simulations of SB treatment only and simulations of SB treatmet plus WRA treatment using the Wilcoxon signed-rank test. The alternative hypothesis is that the prevalence of M\&HI infections in adolescent girls and WRA is less in the scenario with the new treatment guidelines.

\section{Results}

\section{Moderate-prevalence settings}

The ICL model predicts that with ongoing annual SB treatment the prevalence of M\&HI infections is eliminated in the women followed in the cohort model by age 25 years, if WRA themselves are not treated, and by age 20 years, if treatment of WRA is implemented (Figure 1, Table 2). For semi-annual treatment, the decline in M\&HI infections is even faster (Figure 2, Table 3). This is because in the ICL model, adults and children have similar infection levels to begin with (i.e. they are considered to contribute and be exposed to transmission equally). As a result, the Fol acting on the girls/women decreases markedly over time due to the reduced infection levels and thus contribution of pSAC/SAC to transmission.

In contrast, in the EMC model the reduction in the Fol from SB treatment is much less pronounced. A reduction of the Fol occurs over the first ten years after which a new equilibrium Fol under treatment is reached. The reason is that in the EMC model, pre-control infection levels in children are lower than those of adults (i.e. they are considered to contribute and be exposed to transmission differentially). The EMC model predicts a reduction of $23.0 \%(95 \% \mathrm{Cl}: 20.1-26.0 \%)$ in the average point prevalence of women aged 15-50 years where annual SB treatment is ongoing (Figure 1, Table 2) and a reduction of $23.0 \%$ (95\%Cl: $20.5-25.7 \%$ ) where semi-annual SB is ongoing (Figure 2, Table 3). The predicted relative reduction in the average point prevalence of adolescent girls aged $15-19$ years is greater, $55.2 \%(95 \% \mathrm{Cl}$ : $50.6-60.3 \%)$ where annual SB treatment is implemented and $53.0 \%(95 \% \mathrm{Cl}: 48.0-58.6 \%)$ where semiannual SB treatment is implemented. For women aged 20-50 years, the EMC model predicts a relative reduction by treatment during pregnancy of lactation in the average point prevalence of M\&HI infections of $18.3 \%$ (95\%Cl: $15.0-21.7 \%)$ where annual SB treatment is implemented and of $19.0 \%(95 \% \mathrm{Cl}: 16.2-$ 21.9\%) where semi-annual SB treatment is implemented (Figures 1 and 2, Tables 2 and 3).

\section{High-prevalence settings}

The ICL model predicts a relative reduction in the average prevalence of M\&HI infection in WRA aged 1550 years of $5.11 \%$ (95\% Cl: $2.03-7.62 \%$ ) with ongoing semi-annual SB treatment (Figure 3, Table 4). For 
adolescent girls (15-19 years), this reduction was $16.8 \%(95 \% \mathrm{Cl}: 10.7-23.6 \%)$, while for women aged $20-$ 50 years it was $3.17 \%(95 \% \mathrm{Cl}: 0.30-5.70 \%)$.

The EMC model predicts a relative reduction in the average point prevalence of WRA (15-50 years) of $13.1 \%(95 \% \mathrm{Cl}: 11.6-14.6 \%)$ with ongoing SB treatment. The relative reduction in the average prevalence of $\mathrm{M} \& \mathrm{HI}$ infections in adolescent girls and in women aged $20-50$ years is $30.8 \%$ (95\% Cl: $27.3-$ $34.5 \%$ )and $10.4 \%$ (95\% Cl: $8.52-12.2 \%$ ), respectively, according to the EMC model (Figure 3, Table 4). The results for the cohort model simulations are summarised in Figure 4.

\section{Discussion}

The results obtained from the cohort model using Fol from two different individual-based stochastic models of STH transmission suggest that annual deworming treatment of adolescent girls reduces the prevalence of $\mathrm{M} \& \mathrm{HI}$ infections in this age group by up to $60 \%$ (EMC model) in moderate transmission settings and by $12-27 \%$ (both EMC and ICL models) in high-transmission settings. The reductions in moderate transmission settings predicted by the ICL model are highly variable. Overall the reductions are comparable to the impact of SB treatment of pSAC/SAC. The results also suggest that treatment of WRA during pregnancy and lactation reduces the prevalence of $M \& H I$ infections by a small but significant fraction of less than $20 \%$. In moderate prevalence settings where infection levels are uniformly distributed over age (ICL model), reductions of the Fol over time achieved by ongoing SB treatment alone can prevent morbidity in WRA, and consequently deworming of WRA is not necessary. However, for highly endemic settings (both models) and settings where adults host the majority of the hookworm population (EMC model), morbidity in WRA will persist at low levels during SB treatment, which allows for (some) additional benefit of targeted deworming of WRA.

The lower impact of treatment during pregnancy and lactation on the prevalence of M\&HI infections compared to annual treatment during adolescence or SB treatment is expected, as it corresponds to lowcoverage treatment in adult women. Assuming on average four pregnancies per woman, each woman receives treatment on average every seven to eight years. This frequency is unlikely to completely prevent re-infection and the re-establishment of M\&HI infections between treatments.

The differences in the predictions between the two models can be primarily explained by the different age-intensity profiles of infection in the two models and different assumptions about the form of density dependent egg production by adult female worms. The same factors also explain the differences in baseline prevalences of M\&HI infections between the two models (green line in Figures 1-3). The ICL model assumes that exposure to infection is constant over all age groups, such that the worm burden rises to a plateau as people age, as observed in the recent Tumikia study data from Kenya [16]. In contrast, the EMC model assumes that exposure and contribution are 0 at age 0 months, increase linearly up to age ten and stay constant thereafter. This produces a pattern of infection intensity that increases with age up to the age of about 15 , matching some previously published age-intensity profiles from 
various countries [8]. Accordingly, SB treatment has a greater impact on the Fol in the ICL model compared to the EMC model in moderate-transmission settings.

A second explanation for the differences between predictions of the two models relates to density dependence in female worm fecundity, which describes how the egg production per female worm declines as the number of worms in a host increases [18]. The EMC model assumes a hyperbolic saturation of the density-dependent fecundity of female worms inside the host, while the ICL model assumes the density-dependent fecundity of female worms decays in an exponential manner with negative exponent to almost zero as worm burdens rise to high levels [18]. The two functions are similar for low and moderate worm burdens, but different for high worm burdens (eggs produced per female worm decline more rapidly with exponential saturation). As a result, in individuals with high worm burden, female hookworm produce more eggs in the EMC model than in the ICL model. Consequently, killing the same number of worms by treatment leads to a greater reduction in epg in the EMC model compared to the ICL model. Moreover, because in the ICL model density-dependent processes lead to a reduction in the total number of eggs produced inside a host when worm burdens are very high, a reduction in the worm burden following treatment (and re-infection) can result in higher egg production inside a host. This explains why the impact on the prevalence of M\&HI infections in high-transmission settings is less in the ICL model than in the EMC model.

In the simulations, we assumed effective treatment coverage of $75 \%$ and random-access to treatment. Not all countries may achieve this, and non-access to treatment is likely non-random in reality. However, here our focus is on comparing two different treatment strategies. Provided that all assumptions are the same for simulations of the different scenarios that we compare, the results will be informative with respect to the treatment strategies.

When girls/women stop receiving regular SB MDA at effective coverage each year (at age 14 years according to the old guidelines, at age 18 according to the new guidelines), the prevalence of any infection and the prevalence of M\&HI infection are expected to be low in the cohort directly after treatment stops. How long resurgence of prevalences to endemic equilibrium levels will take depends on the worm life expectancy (two or three years in the case of hookworm) and whether or not treatment has lowered the prevalence of any infection so much that it is close to the transmission breakpoint. The transmission breakpoint is the prevalence below which transmission of the parasite cannot be sustained and the parasite population becomes extinct. If the latter has been achieved, models predict that resurgence of infection can take many years and to depend on people movements reintroducing infective stages into the environments where transmission interruption has been achieved [10, 19]. If the prevalence of any infection is not close to the transmission breakpoint after treatment, the time to resurgence of infection in the population will be about equivalent to the worm life expectancy, i.e. 2-3 years. In the cohort model, we only look at the prevalence of infection in a cohort of girls/women and not at the background population that they are embedded in. Consequently, we cannot conclude from the results of the cohort model on its own if the transmission breakpoint has been reached or not. In the absence of any treatment (SB treatment or treatment of WRA), the prevalence and the prevalence of M\&HI 
infections in the cohort at any age is always higher than if treatment at effective coverage is implemented (Supplementary Figure 1 in Additional File 2).

If treatment has not eliminated hookworm transmission, there will be a Fol that causes a resurgence of infection between treatments, but not back to the endemic equilibrium levels that would be observed in the absence of treatment (compare spikes between treatments and equilibrium prevalence of M\&HI infection in Figures 1-3). Resurgence between treatments does not exclude reaching the target of an MDA programme (e.g. $<2 \% \mathrm{M} \& H I$ infections). However, the treatment strategy must be intensive enough for a given intrinsic transmission intensity in a defined setting and must be applied for long enough to reach the target. This has been shown in previous simulation studies [11, 20].

Resurgence of infection may still occur quickly in locations where transmission intensity is high and the infectious reservoir has not been sustainably depleted. For example, Ortu et al. 2016 report that a single year of missed treatment led STH resurgence back to baseline levels in an MDA programme for STH in Burundi where the dominant species was $A$. lumbricoides [21]. In a systematic review and meta-analysis of re-infection with STH after MDA, Jia et al. 2012 found that re-infection between treatments occurs quickly. Twelve months after the last treatment prevalence resurged to $94 \%$ of baseline levels in $A$. lumbricoides, $82 \%$ of baseline levels in T. trichiura and $56 \%$ of baseline levels in hookworm. Therefore, Jia et al. recommended frequent antihelminthic treatment [22]. Similarly, Gunawardena et al. 2011 found a prevalence of M\&HI infections of 11.6\% four years after MDA ceased [23]. Appleton et al. 2009 reported rapid resurgence to baseline prevalence levels four to twelve months after treatment in slum-dwelling children in South Africa [24].

Another study that shows that M\&HI infections can be acquired rapidly is Menzies et al. 2014. The study, set in Ecuador, investigated how quickly children are infected with STH during the first three years of life. The authors found that prevalence of any STH infection was about $25 \%$ at three years of age and that 10 $15 \%$ of infected three-year-old children had M\&HI infections [25]. This is accordance with the prevalence of M\&HI infections a few years after treatment in our simulations.

However, rapid resurgence at the local level does not necessarily contradict sustained low prevalences and reaching the WHO 2030 morbidity target at the country level. At the country level many locations, especially those with low-moderate baseline prevalences, could successfully reach morbidity control, and prevalences could be brought down to levels near the transmission breakpoint from where resurgence, if it happens, takes longer [19]. If the baseline survey happened many years in the past, it is also likely that economic development has occurred in a country and reduced the transmission intensity in many locations, which is another explanation why resurgence to baseline levels is not observed at the country level.

\section{Conclusion}

Overall, our results suggest that treatment of WRA can achieve a small but significant decrease in morbidity and this can be obtained at very low cost: distribution cost will be near to zero and he cost of 
the tablets is of few cents a dose. Annual treatment adolescent girls during HPV vaccine programmes has a similar impact on morbidity as deworming of pSAC/SAC in this age group. Ongoing SB treatment in combination with treatment of WRA may reduce morbidity in WRA, particularly in highly endemic areas and areas where infection levels are concentrated in adults. We therefore recommend that the impact of treating WRA on morbidity in this demographic should be monitored. Regardless of the population-level effectiveness of treating WRA, deworming is still expected to be beneficial to pregnant women and their children: STH infection of mothers during pregnancy has been shown to correlate with an increased risk of anaemia which increases the risk of pregnancy complications $[3,26]$ and deworming in antenatal cliniccs demonstrated to reverse this [27]. In addition, antenatal treatment increases birthweight and reduces mortality $[28,29]$ and the risk of STH infection in very young children (0-3 years) [25]. At this young age, STH infection can have a severe impact on child development.

\section{Abbreviations}

MDA: mass drug administration, M\&HI infections: moderate- and high-intensity infections, pSAC: preschool-age children, SAC: school-age children, SB: school-based, STH: soil-transmitted helminths, WHO: World Health Organization, WRA: women of reproductive age

\section{Declarations}

\section{Ethics approval and consent to participate}

Not applicable.

\section{Consent for publication}

Not applicable.

\section{Availability of data and materials}

Data sharing not applicable to this article as no datasets were generated or analysed during the current study. The code that has been used for the simulation study has been published and can be accessed at https://github.com/caro-veg/STH_WRA_Morbidity.

\section{Competing interests}

The authors declare no competing interests

\section{Funding}

The work was funded by the Bill and Melinda Gates Foundation in partnership with the Task Force for Global Health through the NTD Modelling Consortium [OPP105116 and OPP108566]. CV, SB, RJH, JET, RMA are supported by the UK Medical Research Council (MRC) and the UK Department for International 
Development (DFID) under the MRC/DFID Concordat agreement which is also part of the EDCTP2 programme supported by the European Union. JET, RJH, RMA were supported by the Bill and Melinda Gates Foundation via the DeWorm3 (OPP1129535) award to the Natural History Museum in London. LEC further acknowledges funding from the Dutch Society for Scientific Research [NWO, grant 016.Veni.178.023]. FG acknowledges funding from a European Marie Skłodowska-Curie fellowship [H2020-COFUND-2015-FP-707404]. The funders had no role in study design, data collection and analysis, decision to publish, or preparation of the manuscript.

\section{Authors' contributions}

AM conceived the original study. LC, SdV, RMA, AM, JET, RJH, FG, CV designed the study. CV, FG, SB, VM carried out the analysis. CV wrote the original manuscript All authors contributed to editing and revising the manuscript.

\section{Acknowledgements}

Not applicable.

\section{References}

1. World Health Organization: Guideline: Preventive chemotherapy to control soil-transmitted helminth infections in at-risk population groups. Geneva: World Health Organization; 2017.

2. Lwambo NJ, Bundy DA, Medley GF. A new approach to morbidity risk assessment in hookworm endemic communities. Epidemiol Infect. 1992;108:469-81.

3. Brooker S, Hotez PJ, Bundy DAP. Hookworm-related anaemia among pregnant women: A systematic review. PLoS Negl Trop Dis. 2008;2:e291.

4. World Health Organization: Soil-transmitted helminthiases: Eliminating soil-transmitted helminthiases as a public health problem in children: Progress report 2001-2010 and strategic plan 2011-2020. Geneva: World Health Organization; 2012.

5. Insights from quantitative analysis and mathematical modelling on the proposed who 2030 goals for soil-transmitted helminths. Gates Open Res. 2019;3:1632.

6. Marocco C, Bangert M, Joseph SA, Fitzpatrick C, Montresor A. Preventive chemotherapy in one year reduces by over $80 \%$ the number of individuals with soil-transmitted helminthiases causing morbidity: Results from meta-analysis. Trans R Soc Trop Med Hyg. 2017;111:12-7.

7. Anderson RE, May RM. Infectious diseases of humans: Dynamics and control. Oxford Science Publications. 1991.

8. Brooker S, Bethony J, Hotez PJ. Human hookworm infection in the 21st century. Adv Parasitol. 2004;58:197-288.

9. Truscott JE, Turner HC, Farrell SH, Anderson RM. Soil-transmitted helminths: Mathematical models of transmission, the impact of mass drug administration and transmission elimination criteria. Adv 
Parasitol. 2016;94:133-98.

10. Truscott JE, Werkman M, Wright JE, Farrell SH, Sarkar R, Asbjornsdottir K, et al. Identifying optimal threshold statistics for elimination of hookworm using a stochastic simulation model. Parasit Vectors. 2017;10:321.

11. Coffeng LE, Bakker R, Montresor A, de Vlas SJ. Feasibility of controlling hookworm infection through preventive chemotherapy: A simulation study using the individual-based wormsim modelling framework. Parasit Vectors. 2015;8:541.

12. The World Bank: Fertility rate, total (births per woman). https://data.worldbank.org/indicator/SP.DYN.TFRT.IN (2020). Accessed 19/08/2020 2020.

13. Bangert M, Bancalari P, Mupfasoni D, Mikhailov A, Gabrielli AF, Montresor A. Provision of deworming intervention to pregnant women by antenatal services in countries endemic for soil-transmitted helminthiasis. PLoS Negl Trop Dis. 2019;13:e0007406.

14. Coffeng LE, Truscott JE, Farrell SH, Turner HC, Sarkar R, Kang G, et al. Comparison and validation of two mathematical models for the impact of mass drug administration on ascaris lumbricoides and hookworm infection. Epidemics. 2017;18:38-47.

15. Central Bureau of Statistics CBSK, Ministry of Health MOHK, Macro ORC: Kenya demographic and health survey 2003. Calverton, Maryland, USA: CBS, MOH, and ORC Macro; 2004.

16. Truscott JE, Ower AK, Werkman M, Halliday K, Oswald WE, Gichuki PM, et al. Heterogeneity in transmission parameters of hookworm infection within the baseline data from the tumikia study in kenya. Parasit Vectors. 2019;12:442.

17. Elkins DB, Haswell-Elkins M, Anderson RM. The epidemiology and control of intestinal helminths in the pulicat lake region of southern india. I. Study design and pre- and post-treatment observations on ascaris lumbricoides infection. Trans R Soc Trop Med Hyg. 1986;80:774-92.

18. Anderson RM, May RM. Helminth infections of humans: Mathematical models, population dynamics, and control. Adv Parasitol. 1985;24:1-101.

19. Hardwick RJ, Vegvari C, Truscott JE, Anderson RM. The 'breakpoint' of soil-transmitted helminths with infected human migration. J Theor Biol. 2020;486:110076.

20. Anderson R, Truscott J, Hollingsworth TD. The coverage and frequency of mass drug administration required to eliminate persistent transmission of soil-transmitted helminths. Philos Trans $R$ Soc Lond B Biol Sci. 2014;369:20130435.

21. Ortu G, Assoum M, Wittmann U, Knowles S, Clements M, Ndayishimiye O, et al. The impact of an 8year mass drug administration programme on prevalence, intensity and co-infections of soiltransmitted helminthiases in burundi. Parasit Vectors. 2016;9:513.

22. Jia TW, Melville S, Utzinger J, King CH, Zhou XN. Soil-transmitted helminth reinfection after drug treatment: A systematic review and meta-analysis. PLoS Negl Trop Dis. 2012;6:e1621.

23. Gunawardena K, Kumarendran B, Ebenezer R, Gunasingha MS, Pathmeswaran A, de Silva N. Soiltransmitted helminth infections among plantation sector schoolchildren in sri lanka: Prevalence after ten years of preventive chemotherapy. PLoS Negl Trop Dis. 2011;5:e1341-e. 
24. Appleton CC, Mosala TI, Levin J, Olsen A. Geohelminth infection and re-infection after chemotherapy among slum-dwelling children in durban, south africa. Ann Trop Med Parasitol. 2009;103:249-61.

25. Menzies SK, Rodriguez A, Chico M, Sandoval C, Broncano N, Guadalupe I, et al. Risk factors for soiltransmitted helminth infections during the first 3 years of life in the tropics; findings from a birth cohort. PLoS Negl Trop Dis. 2014;8:e2718-e.

26. Mahande AM, Mahande MJ. Prevalence of parasitic infections and associations with pregnancy complications and outcomes in northern tanzania: A registry-based cross-sectional study. BMC Infectious Diseases. 2016;16:78.

27. Torlesse $\mathrm{H}$, Hodges $\mathrm{M}$. Anthelminthic treatment and haemoglobin concentrations during pregnancy. Lancet. 2000;356:1083.

28. Christian P, Khatry SK, West KP, Jr. Antenatal anthelmintic treatment, birthweight, and infant survival in rural nepal. Lancet. 2004;364:981-3.

29. Passerini L, Casey GJ, Biggs BA, Cong DT, Phu LB, Phuc TQ, et al. Increased birth weight associated with regular pre-pregnancy deworming and weekly iron-folic acid supplementation for vietnamese women. PLoS Negl Trop Dis. 2012;6:e1608.

\section{Tables}

Table 1: Overview of simulated treatment scenarios. For each baseline prevalence and treatment frequency we compare the currently implemented treatment strategy (SB treatment only) with the new recommended treatment strategy (SB treatment \& treatment of WRA). All analyses were done separately with the Fols extracted from the EMC and ICL models.

\begin{tabular}{lll}
\hline $\begin{array}{l}\text { Baseline prevalence of any } \\
\text { hookworm infection }\end{array}$ & SB treatment only & SB treatment \& treatment of \\
& & WRA \\
\hline Moderate & Annual SB treatment & Annual SB treatment \\
& Semi-annual SB treatment & Semi-annual SB treatment \\
High & Semi-annual SB treatment & Semi-annual SB treatment \\
\hline
\end{tabular}


Table 2: Average prevalences of M\&HI infections in WRA (\%). Moderate baseline prevalence (20$50 \%)$, annual SB treatment. The Fol inputted into the cohort model was extracted from ICL and EMC fully stochastic individual-based models with annual ongoing SB treatment, i.e. Fol decreased over time. In the ICL model prevalences of M\&HI infections in WRA are well below $1 \%$. Therefore, the reduction in the prevalence of $M \& H I$ infections in absolute numbers is negligible and the uncertainty large.

\begin{tabular}{|c|c|c|c|c|c|}
\hline & Age period & $\begin{array}{l}\text { SB only [mean } \\
\text { (95\% credible } \\
\text { interval)] }\end{array}$ & $\begin{array}{l}\text { SB + WRA [mean } \\
\text { ( } 95 \% \text { credible } \\
\text { interval)] }\end{array}$ & $\begin{array}{l}\text { \% reduction } \\
\text { [mean ( } 95 \% \\
\text { credible } \\
\text { interval)] }\end{array}$ & p-value \\
\hline $\mathrm{ICL}$ & $\begin{array}{l}15-50 \\
\text { years }\end{array}$ & $\begin{array}{l}0.0216(0.0142- \\
0.0328)\end{array}$ & $\begin{array}{l}4.81 \times 10^{-3}(2.35 \mathrm{x} \\
\left.10^{-3}-8.57 \times 10^{-3}\right)\end{array}$ & $77.8(59.2-91.9)$ & $<2.2 \times 10^{-16}$ \\
\hline $\mathrm{ICL}$ & $\begin{array}{l}15-19 \\
\text { years }\end{array}$ & $\begin{array}{l}0.101(0.06- \\
0.141)\end{array}$ & $\begin{array}{l}0.0173(3.33 \times 10 \\
\left.3^{3}-0.03\right)\end{array}$ & $83.5(66.7-95.9)$ & $<2.2 \times 10^{-16}$ \\
\hline $\mathrm{ICL}$ & $\begin{array}{l}20-50 \\
\text { years }\end{array}$ & $\begin{array}{l}8.828 \times 10^{-3}(3.88 \\
\left.\times 10^{-3}-0.0127\right)\end{array}$ & $\begin{array}{l}2.73 \times 10^{-3}(5.54 \times \\
\left.10^{-4}-5.57 \times 10^{-3}\right)\end{array}$ & $61.5(-22.6-100)$ & $<2.2 \times 10^{-16}$ \\
\hline EMC & $\begin{array}{l}15-50 \\
\text { years }\end{array}$ & $5.06(4.54-5.46)$ & $3.90(3.47-4.26)$ & $23.0(20.1-26.0)$ & $<2.2 \times 10^{-16}$ \\
\hline EMC & $\begin{array}{l}15-19 \\
\text { years }\end{array}$ & $4.42(3.93-4.85)$ & $2.00(1.70-2.35)$ & $55.2(50.6-60.3)$ & $<2.2 \times 10^{-16}$ \\
\hline EMC & $\begin{array}{l}20-50 \\
\text { years }\end{array}$ & $5.16(4.66-5.59)$ & $4.21(3.78-4.59)$ & $18.3(15.0-21.7)$ & $<2.2 \times 10^{-16}$ \\
\hline
\end{tabular}

\footnotetext{
${ }^{1}$ The relative reduction is only calculated from time points where the prevalence of $M \& H I$ infections in simulations with the old treatment strategy (SB only) is greater than 0 . All time points were used in the statistical comparison.
} 
Table 3: Average prevalences of M\&HI infections in WRA (\%). Moderate baseline prevalence (2050\%), semi-annual SB treatment. The Fol inputted into the cohort model was extracted from ICL and EMC fully stochastic individual-based models with ongoing semi-annual SB treatment, i.e. Fol decreased over time. In the ICL model prevalences of M\&HI infections in WRA are below $1 \%$. Therefore, the reduction in the prevalence of $M \& H I$ infections in absolute numbers is negligible and the uncertainty large.

\begin{tabular}{|c|c|c|c|c|c|}
\hline & Age period & $\begin{array}{l}\text { SB only [mean } \\
\text { (95\% credible } \\
\text { interval)] }\end{array}$ & $\begin{array}{l}\text { SB + WRA [mean } \\
\text { (95\% credible } \\
\text { interval)] }\end{array}$ & $\begin{array}{l}\text { \% reduction } \\
\text { [mean ( } 95 \% \\
\text { credible } \\
\text { interval)] }^{1}\end{array}$ & p-value \\
\hline $\mathrm{ICL}$ & $\begin{array}{l}15-50 \\
\text { years }\end{array}$ & $\begin{array}{l}3.42 \times 10^{-4}(0- \\
\left.1.43 \times 10^{-3}\right)\end{array}$ & $\begin{array}{l}5.23 \times 10^{-5}(0- \\
\left.4.75 \times 10^{-4}\right)\end{array}$ & $41(0-100)$ & $9.33 \times 10^{-7}$ \\
\hline $\mathrm{ICL}$ & $\begin{array}{l}15-19 \\
\text { years }\end{array}$ & $\begin{array}{l}1.97 \times 10^{-3}(0- \\
0.01)\end{array}$ & $\begin{array}{l}3.33 \times 10^{-4}(0- \\
\left.3.33 \times 10^{-3}\right)\end{array}$ & $37(0-100)$ & $1.27 \times 10^{-6}$ \\
\hline $\mathrm{ICL}$ & $\begin{array}{l}20-50 \\
\text { years }\end{array}$ & $0(0-0)$ & $0(0-0)$ & $0(0-0)$ & NA \\
\hline EMC & $\begin{array}{l}15-50 \\
\text { years }\end{array}$ & $4.56(4.11-4.97)$ & $3.51(3.13-3.85)$ & $23.0(20.5-25.7)$ & $<2.2 \times 10^{-16}$ \\
\hline EMC & $\begin{array}{l}15-19 \\
\text { years }\end{array}$ & $3.72(3.29-4.11)$ & $1.75(1.50-2.01)$ & $53.0(48.0-58.6)$ & $<2.2 \times 10^{-16}$ \\
\hline EMC & $\begin{array}{l}20-50 \\
\text { years }\end{array}$ & $4.70(4.25-5.10)$ & $3.81(3.41-4.17)$ & $19.0(16.2-21.9)$ & $<2.2 \times 10^{-16}$ \\
\hline
\end{tabular}

${ }^{1}$ The relative reduction is only calculated from time points where the prevalence of $\mathrm{M} \& \mathrm{HI}$ infections in simulations with the old treatment strategy (SB only) is greater than 0 . All time points were used in the statistical comparison. 
Table 4: Average prevalences of M\&HI infections in WRA (\%). High baseline prevalence (>50\%) The Fol inputted into the cohort model was extracted from ICL and EMC fully stochastic individual-based models with ongoing SB treatment, i.e. Fol decreased over time.

\begin{tabular}{|c|c|c|c|c|c|}
\hline & Age period & $\begin{array}{l}\text { SB only [mean } \\
\text { (95\% credible } \\
\text { interval)] }\end{array}$ & $\begin{array}{l}\text { SB + WRA [mean } \\
\text { (95\% credible } \\
\text { interval)] }\end{array}$ & $\begin{array}{l}\text { \% reduction } \\
\text { [mean (95\% } \\
\text { credible } \\
\text { interval)] }\end{array}$ & p-value \\
\hline $\mathrm{ICL}$ & $\begin{array}{l}15-50 \\
\text { years }\end{array}$ & $8.24(7.72-8.79)$ & $7.82(7.35-8.30)$ & $5.11(2.03-7.62)$ & $<2.2 \times 10^{-16}$ \\
\hline $\mathrm{ICL}$ & $\begin{array}{l}15-19 \\
\text { years }\end{array}$ & $8.20(7.56-8.80)$ & $6.82(6.26-7.35)$ & $16.8(10.7-23.6)$ & $<2.2 \times 10^{-16}$ \\
\hline $\mathrm{ICL}$ & $\begin{array}{l}20-50 \\
\text { years }\end{array}$ & $8.25(7.77-8.82)$ & $7.99(7.50-8.48)$ & $3.17(0.30-5.70)$ & $<2.2 \times 10^{-16}$ \\
\hline EMC & $\begin{array}{l}15-50 \\
\text { years }\end{array}$ & $11.5(10.8-12.2)$ & $10.0(9.29-10.6)$ & $13.1(11.6-14.6)$ & $<2.2 \times 10^{-16}$ \\
\hline EMC & $\begin{array}{l}15-19 \\
\text { years }\end{array}$ & $10.5(9.77-11.2)$ & $7.29(6.60-7.88)$ & $30.8(27.3-34.5)$ & $<2.2 \times 10^{-16}$ \\
\hline EMC & $\begin{array}{l}20-50 \\
\text { years }\end{array}$ & $11.7(11.0-12.3)$ & $10.5(9.77-11.1)$ & $10.4(8.52-12.2)$ & $<2.2 \times 10^{-16}$ \\
\hline
\end{tabular}

Figures 

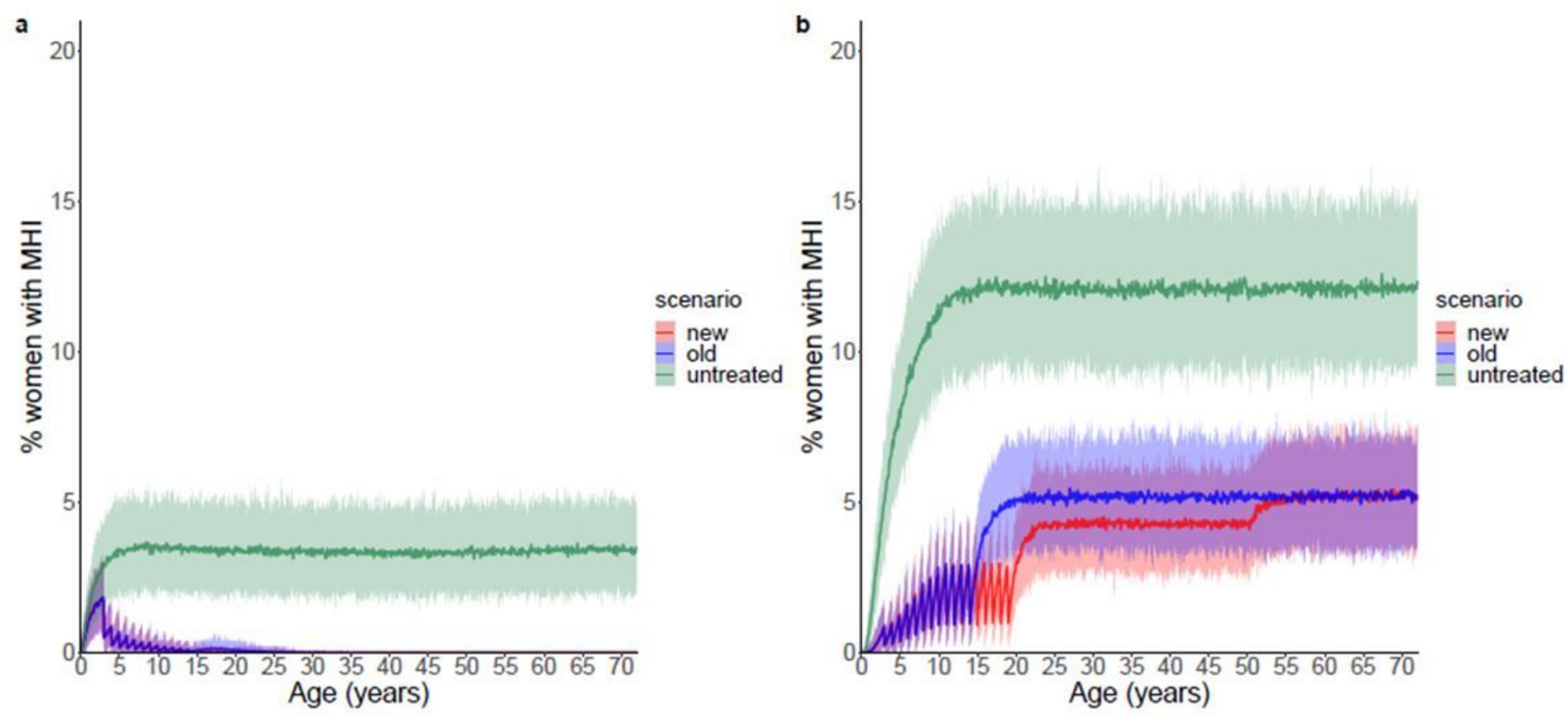

\section{Figure 1}

Percent of girls/women with M\&HI infections as they age. Moderate baseline prevalence (20-50\%), annual SB treatment. The Fol inputted into the cohort model was extracted from ICL and EMC fully stochastic individual-based models with ongoing SB treatment, i.e. Fol decreased over time. a: Imperial College London (ICL) results. b: Erasmus MC (EMC) results. Solid lines: mean; pale area: 95\% credible interval. Green: no treatment. Blue: treatment of pSAC and SAC only (old guidelines). Red: treatment of pSAC and SAC plus annual treatment of adolescent girls (15-19 years) plus treatment of women during pregnancy and lactation (new suggested guidelines). 

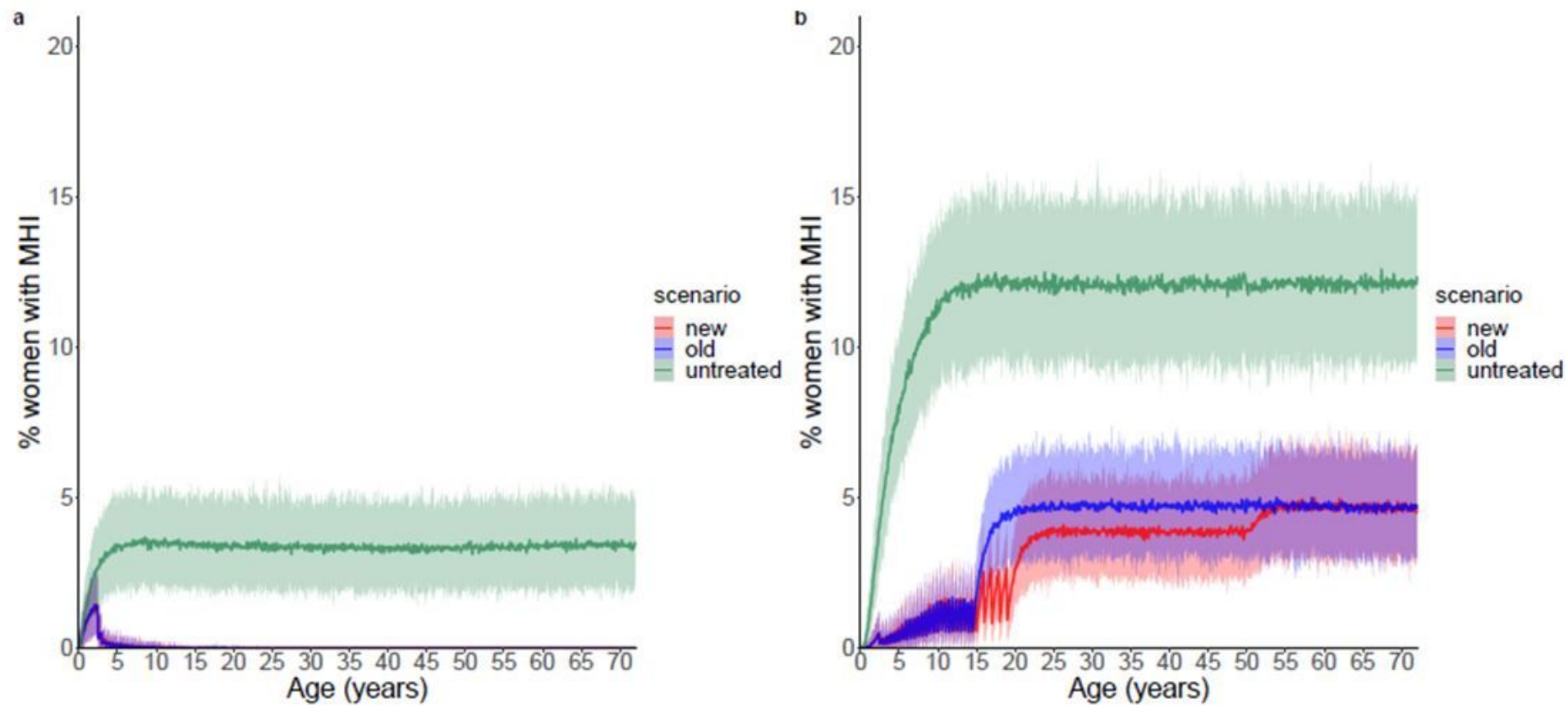

Figure 2

Percent of girls/women with M\&HI infections as they age. Moderate baseline prevalence (20-50\%), semiannual SB treatment. The Fol inputted into the cohort model was extracted from ICL and EMC fully stochastic individual-based models with ongoing SB treatment, i.e. Fol decreased over time. a: Imperial College London (ICL) results. b: Erasmus MC (EMC) results. Solid lines: mean; pale area: $95 \%$ credible interval. Green: no treatment. Blue: treatment of pSAC and SAC only (old guidelines). Red: treatment of pSAC and SAC plus annual treatment of adolescent girls (15-19 years) plus treatment of women during pregnancy and lactation (new suggested guidelines).
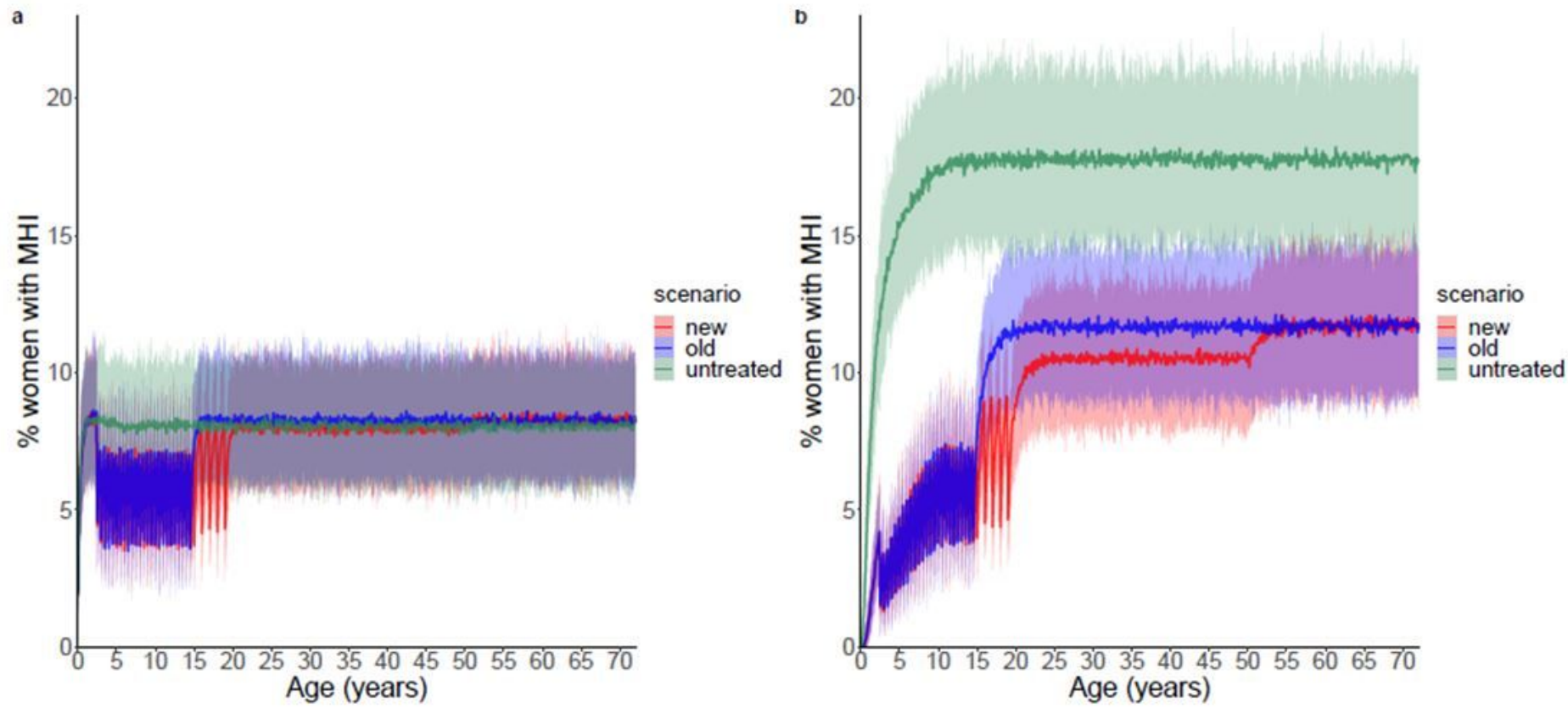
Figure 3

Percent of girls/women with M\&HI infections as they age. High baseline prevalence ( $>50 \%)$, semi-annual SB treatment. The Fol inputted into the cohort model was extracted from ICL and EMC fully stochastic individual-based models with ongoing SB treatment, i.e. Fol decreased over time. a: Imperial College London (ICL) results. b: Erasmus MC (EMC) results. Solid lines: mean; pale area: 95\% credible interval. Green: no treatment. Blue: treatment of pSAC and SAC only (old guidelines). Red: treatment of pSAC and SAC plus annual treatment of adolescent girls (15-19 years) plus treatment of women during pregnancy and lactation (new suggested guidelines).

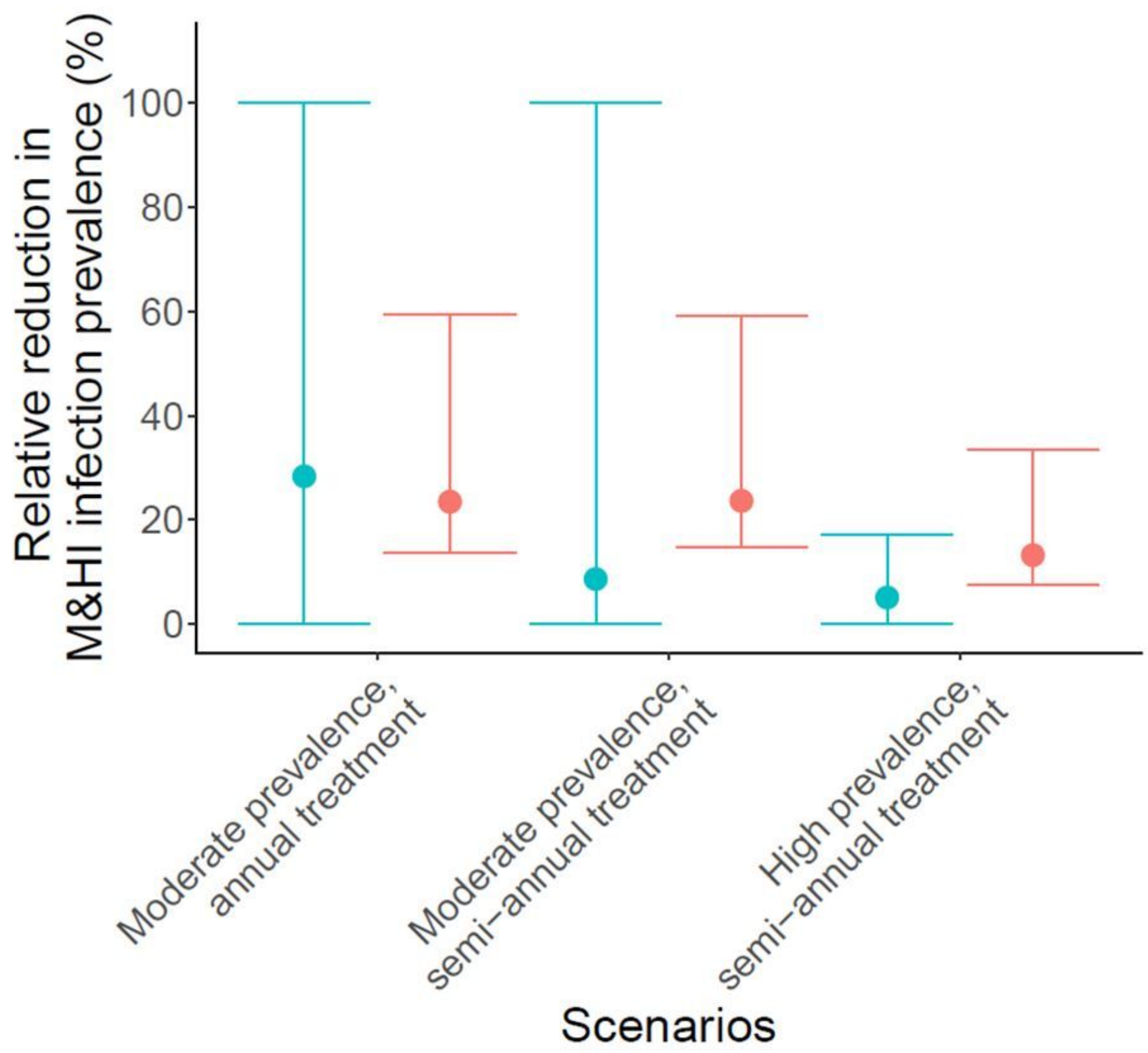

$E M C \bullet I C L$

Figure 4 
Relative reduction in the prevalence (in \%) of M\&HI infections in WRA (15-50 years) with the new treatment strategy (treating school-aged children plus treating adolescent girls annually, treating women twice during pregnancy and once during lactation) compared to the prevalence of M\&HI infections in WRA with the old treatment strategy (treating only school-aged children, no treatment of WRA). The Fol inputted into the cohort model was extracted from ICL and EMC fully stochastic individual-based models with ongoing SB treatment, i.e. Fol decreased over time. Results were obtained from 100 iterations of a cohort model of 500 women run for each scenario. Treatment frequency depended on the transmission setting, according to WHO guidelines. Error bars represent 95\% credible intervals. EMC: Erasmus MC, ICL: Imperial College London.

\section{Supplementary Files}

This is a list of supplementary files associated with this preprint. Click to download.

- Additionalfile1.pdf

- Additionalfile2.pdf 